\title{
Compton Heating of the Intergalactic Medium by the Hard X-ray Background
}

\author{
Piero Madau ${ }^{1}$ \\ Space Telescope Science Institute, 3700 San Martin Drive, Baltimore, MD 21218 \\ and \\ George Efstathiou \\ Institute of Astronomy, Madingley Road, Cambridge CB3 0HA, UK
}

\begin{abstract}
High-resolution hydrodynamics simulations of the Ly $\alpha$ forest in cold dark matter dominated cosmologies appear to predict line-widths that are substantially narrower than those observed. Here we point out that Compton heating of the intergalactic gas by the hard X-ray background (XRB), an effect neglected in all previous investigations, may help to resolve this discrepancy. The rate of gain in thermal energy by Compton scattering will dominate over the energy input from hydrogen photoionization if the $\mathrm{XRB}$ energy density is $\sim 0.2 x /\langle\epsilon\rangle$ times higher than the energy density of the UV background at a given epoch, where $x$ is the hydrogen neutral fraction in units of $10^{-6}$ and $\langle\epsilon\rangle$ is the mean $\mathrm{X}$-ray photon energy in units of $m_{e} c^{2}$. The numerical integration of the time-dependent rate equations shows that the intergalactic medium approaches a temperature of about $1.5 \times 10^{4} \mathrm{~K}$ at $z>3$ in popular models for the redshift evolution of the extragalactic background radiation. The importance of Compton heating can be tested experimentally by measuring the Ly $\alpha$ line-width distribution as a function of redshift, thus the Ly $\alpha$ forest may provide a useful probe of the evolution of the XRB at high redshifts.
\end{abstract}

Subject headings: cosmology: theory - diffuse radiation - intergalactic medium - quasars: absorption lines - X-rays: general

\section{Introduction}

Neutral hydrogen in the intergalactic medium (IGM) produces a plethora of Ly $\alpha$ absorption lines in the spectra of high redshift quasars. Numerical N-body/hydrodynamics simulations of structure formation in the IGM, within the framework of cold dark matter (CDM) dominated cosmologies (Cen et al. 1994; Zhang, Anninos, \& Norman 1995; Miralda-Escudé et al. 1996; Hernquist

\footnotetext{
${ }^{1}$ also at Institute of Astronomy, Madingley Road, Cambridge CB3 0HA, UK.
} 
et al. 1996; Zhang et al. 1998; Theuns et al. 1998a,b; Davé et al. 1999; Bryan et al. 1999), have recently provided a definite picture for the origin of the Ly $\alpha$ forest, one of an interconnected network of sheets and filaments with virialized systems (halos) located at their points of intersection. At $z>2$, the lowest column density absorbers $\left(N_{\mathrm{HI}} \sim 10^{12} \mathrm{~cm}^{-2}\right)$ arise in this context in the underdense $\left(\rho_{b} / \bar{\rho}_{b}<1\right)$ minivoids between the filaments, absorbers that give rise to moderate column density lines $\left(N_{\mathrm{HI}} \lesssim 10^{14} \mathrm{~cm}^{-2}\right)$ correspond to modestly overdense $\left(1<\rho_{b} / \bar{\rho}_{b}<5\right)$ filaments, and high column density absorbers $\left(N_{\mathrm{HI}} \gtrsim 10^{15} \mathrm{~cm}^{-2}\right)$ arise in highly overdense $\left(\rho_{b} / \bar{\rho}_{b}>10\right)$ structures at the intersection of filaments (Zhang et al. 1998). Most simulations to date assume that the IGM is photoionized and photoheated by a UV background close to that inferred from quasars (as computed by Haardt \& Madau 1996, hereafter HM96). The overall normalization of the column density distribution depends approximately on the parameter $\Omega_{b}^{2} h^{3} / \Gamma$, where $\Gamma$ is the hydrogen ionization rate, $h$ is the Hubble constant in units of $100 \mathrm{~km} \mathrm{~s}^{-1} \mathrm{Mpc}^{-1}$, and $\Omega_{b}$ is the baryon density parameter. The width of the lines, as measured by the $b$ parameter of a Voigt profile, is set by thermal broadening, peculiar velocities, and Hubble expansion across the filaments.

While the first simulations showed good agreement with the observed line statistics, recent detailed studies at higher numerical resolution have revealed a serious conflict with the data: the models predict median values for the $b$ distribution of about $20 \mathrm{~km} \mathrm{~s}^{-1}$ at $z=3$, compared with the observed median of $30 \mathrm{~km} \mathrm{~s}^{-1}$ (Theuns et al. 1998b; Bryan et al. 1999). One possible way to restore the agreement with observations is to increase the temperature of the IGM, adding to the thermal broadening. As Theuns et al. (1999) have pointed out, this may be achieved by increasing the baryon density $\omega_{b} \equiv \Omega_{b} h^{2}$, or by heating the gas for longer by increasing the age of the universe (e.g. by lowering the total matter density $\Omega_{m}$ ). However, the gas temperature at late times is determined (approximately) by the balance between adiabatic cooling and photoelectric heating and varies as $T \propto\left(\Omega_{b} h / \sqrt{\Omega_{m}}\right)^{1 / 1.7}$ (Hui \& Gnedin 1997). Thus the needed increase in temperature (a factor of 2 ) is difficult to accomplish without conflicting with big bang nucleosynthesis for reasonable values of $\Omega_{m}$ and $h$ (Theuns et al. 1999 and $\S 3$ of this paper). Delaying the reionization of helium may also help (Haehnelt \& Steinmetz 1998), but this appears to boost the temperature by only a small factor. Other mechanisms that could increase the line-width include photoelectric dust heating (Nath, Sethi, \& Shchekinov 1999) and radiative transfer effects during the reionization of helium (Miralda-Escudé \& Rees 1994; Abel \& Haehnelt 1999).

In this Letter we point out that a potentially important heating term has been neglected in all previous numerical studies, namely Compton heating of electrons by hard X-ray background photons. We show that this effect will dominate over photoelectric heating of hydrogen at redshifts $\gtrsim 2$ in popular models for the redshift evolution of the extragalactic background radiation and that it will drive the IGM towards a temperature of about $1.5 \times 10^{4} \mathrm{~K}$ at $z>3$. 


\section{Compton heating of the IGM}

In an IGM that interacts solely with a background of UV ionizing photons (assumed to originate from quasars and/or star-forming galaxies), the dominant heat input is due to photoelectric heating of hydrogen and helium atoms. The energy input from hydrogen photoionization is given by

$$
H_{\mathrm{H}}=4 \pi \bar{n}_{\mathrm{HI}} \int_{\nu_{L}}^{\infty} d \nu \frac{J_{\nu}}{h_{P} \nu} \sigma_{\mathrm{H}}\left(h_{P} \nu-h_{P} \nu_{L}\right),
$$

where $\bar{n}_{\mathrm{H} \text { I }}$ is the mean neutral hydrogen density, $J_{\nu} \propto\left(\nu / \nu_{L}\right)^{-\alpha}$ the specific intensity of the UV radiation background, $\sigma_{\mathrm{H}}=\sigma_{L}\left(\nu / \nu_{L}\right)^{-3}$ the photoionization cross-section, and $h_{P} \nu_{L}$ the energy of the Lyman edge. Equation (11) can be integrated to give

$$
H_{\mathrm{H}}=\bar{n}_{\mathrm{HI}} \sigma_{L} c U_{\mathrm{UV}} \frac{(\alpha-1)}{(\alpha+2)(\alpha+3)}
$$

where $U_{\mathrm{UV}}$ is the (proper) energy density of the metagalactic flux. In a quasar-dominated background, photoheating by He II will be larger than $H_{\mathrm{H}}$ by a factor 1.5-2. The main cooling processes are Compton cooling against the microwave background at high redshifts and adiabatic cooling at redshifts $\lesssim 2$.

Allow now the intergalactic gas to exchange energy with the XRB through Compton heating at a rate

$$
H_{C}=4 \pi \bar{n}_{e} \frac{\sigma_{T}}{m_{e} c^{2}} \int_{0}^{\infty} d \nu J_{\nu}\left(h_{P} \nu-4 k T\right)
$$

where $\bar{n}_{e}$ is the mean electron density, $J_{\nu}$ the specific intensity of the XRB, and $\sigma_{T}$ the Thomson cross-section. Energy is transferred from photons to electrons when $h_{P} \gg \gg 4 k T$, and in this limit equation (3) becomes

$$
H_{C}=\sigma_{T} \bar{n}_{e} c \frac{\left\langle h_{P} \nu\right\rangle}{m_{e} c^{2}} U_{X}
$$

where $\left\langle h_{P} \nu\right\rangle$ is the mean photon energy and $U_{X}$ the energy density of the XRB. For photons above $100 \mathrm{keV}$ relativistic effects become significant, and Compton scattering takes place in the KleinNishina regime. The relativistic generalization of equation (3) can be written as (cf. Blumenthal 1974)

$$
H_{C}=3 \pi \bar{n}_{e} \sigma_{T} \int_{0}^{\infty} d \epsilon J_{\epsilon} \frac{1}{\epsilon^{2}}\left[\frac{\epsilon^{2}-2 \epsilon-3}{2 \epsilon} \ln (1+2 \epsilon)-\frac{10 \epsilon^{4}-51 \epsilon^{3}-93 \epsilon^{2}-51 \epsilon-9}{3(1+2 \epsilon)^{3}}\right]
$$

(for $\epsilon \gg 4 k T / m_{e} c^{2}$ ), where $\epsilon$ is the photon energy in units of $m_{e} c^{2}$. All the energy of the primary electrons will be deposited into the medium as heat (through Coulomb collisions), since energy losses due to collisional excitation and ionization of $\mathrm{H}$ and $\mathrm{He}$ are negligible in such a highly ionized IGM (e.g. Shull \& Van Steenberg 1985).

The XRB spectrum is characterized by a narrow peak in spectral power, $E J_{E}$, at $30 \mathrm{keV}$; a simple analytical fit to the observed energy flux in units of $\mathrm{keV} \mathrm{cm}^{-2} \mathrm{~s}^{-1} \mathrm{keV}^{-1} \mathrm{sr}^{-1}$ yields

$$
J_{E}=7.7 E_{\mathrm{keV}}^{-0.29} \exp \left(-\frac{E_{\mathrm{keV}}}{40}\right)
$$


from 3 to $60 \mathrm{keV}$ (Boldt 1987). At higher energies, the HEAO $1 \mathrm{~A}-4$ data can be fitted with a power-law,

$$
J_{E}=0.26\left(\frac{E_{\mathrm{keV}}}{100}\right)^{-1.75}
$$

from 80 to $400 \mathrm{keV}$ (Kinzer et al. 1997). The preliminary diffuse $\gamma$-ray spectrum from $800 \mathrm{keV}$ to $30 \mathrm{MeV}$ measured with COMPTEL (Kappadath et al. 1996) obeys another power-law,

$$
J_{E}=0.15\left(E_{\mathrm{keV}} / 100\right)^{-1.38} \mathrm{keV} \mathrm{cm}^{-2} \mathrm{~s}^{-1} \mathrm{keV}^{-1} \mathrm{sr}^{-1}
$$

(Kribs \& Rothstein 1997). The total energy density in the $0.003-30 \mathrm{MeV}$ band is $U_{X}=6.3 \times$ $10^{-5} \mathrm{eV} \mathrm{cm}^{-3}$. The mean, "effective" (as it includes a large correction due to Klein-Nishina effects) photon energy is $\left\langle h_{P} \nu\right\rangle=31.8 \mathrm{keV}$. Note that, because of the decline at high energies of the KleinNishina cross-section, the $(\mathrm{X}+\gamma)$-ray heating rate is numerically equivalent to the rate one would derive by assuming pure Thomson scattering of photons in the range $3-180 \mathrm{keV}$ only. With the adopted spectrum, about half of the total energy exchange rate at the present-epoch is provided by photons above $110 \mathrm{keV}$ and about $14 \%$ is contributed by photons above $1 \mathrm{MeV}$.

We can now estimate the relative importance of Compton versus photoelectric heating of hydrogen. The QSO contribution to the local metagalactic flux at the Lyman edge is $J_{L} \approx 10^{-23}$ ergs $\mathrm{cm}^{-2} \mathrm{~s}^{-1} \mathrm{~Hz}^{-1} \mathrm{sr}^{-1}$ (HM96). Assuming a spectral index of $\alpha=1.3$, from equations (1) and (3) we get

$$
\frac{H_{C}}{H_{\mathrm{H}}} \approx 6.8 \times 10^{-7} \frac{\bar{n}_{e}}{\bar{n}_{\mathrm{HI}}}
$$

at $z=0$. The equation of ionization equilibrium for an IGM with mean hydrogen density $\bar{n}_{\mathrm{H}}=1.6 \times 10^{-7}\left(\omega_{b} / 0.019\right) \mathrm{cm}^{-3}$ and temperature $T=2 \times 10^{3} \mathrm{~K}$ gives $\bar{n}_{e} / \bar{n}_{\mathrm{HI}} \sim 1.5 \times 10^{5}$, hence $H_{C} \sim 0.1 H_{\mathrm{H}}$. At the present-epoch, photoelectric heating is then the dominant contribution. At $z=2$, the UV background from optically-selected quasars increases to $J_{L} \approx 3 \times 10^{-22}$ ergs cm ${ }^{-2} \mathrm{~s}^{-1} \mathrm{~Hz}^{-1} \mathrm{sr}^{-1}$ (HM96), the gas temperature reaches $10^{4} \mathrm{~K}$ or higher, and ionization equilibrium now yields $\bar{n}_{e} / \bar{n}_{\mathrm{HI}} \sim 5 \times 10^{5}$. If the bulk of the XRB is produced at high redshifts, $U_{X}$ increases as $(1+z)^{4}$, and the rate of gain in thermal energy per baryon due to Compton scattering goes up as $(1+z)^{4+\beta}$, where $\beta \approx 1 / 3$ is less than unity because Klein-Nishina corrections become more important at high redshifts. The same exercise then yields $H_{C} \sim 1.3 H_{\mathrm{H}}$ at $z=2$.

The relative strength of the two competing heating terms will obviously depend on the redshift evolution of ionizing and X-ray extragalactic radiation. Two cosmological effects may tend to make Compton scattering of hard X-ray photons the dominant source of heating at high redshifts in a highly ionized, low density IGM:

- at fixed ionization state and energy density ratio $U_{X} / U_{\mathrm{UV}}$, the ratio $H_{C} / H_{\mathrm{H}}$ increases with $\left\langle h_{P} \nu\right\rangle$ as $(1+z)^{\beta}$, where $\beta \approx 1 / 3$ for $z \leq 3$;

- at fixed ratio between the quasar UV and X-ray volume emissivities, the radiation energy density $U_{X}$ increases with redshift relative to $U_{\mathrm{UV}}$, as the mean free path of photons at 
$912 \AA$ becomes very small, sources at higher redshifts are severely attenuated, and the UV background is largely "local" beyond a redshift of $z \gtrsim 2$ (e.g. Madau 1992).

In the absence of any other cooling or heating mechanisms, Compton cooling off cosmic microwave background (CMB) photons and Compton heating by the XRB will drive the thermal state of the IGM towards the Compton temperature

$$
T_{C}=\frac{\left\langle h_{P} \nu\right\rangle}{4 k} \frac{U_{X}}{U_{\mathrm{CMB}}}
$$

independently of the electron density. At $z=0, T_{C} \approx 2.2 \times 10^{4} \mathrm{~K}$.

\section{Non-equilibrium models}

In this section we will calculate numerically the thermal history of a uniform IGM of primordial composition within a cosmological context. The code that we use includes the relevant cooling and heating processes (as described in Theuns et al. 1998b, and references therein) and follows the non-equilibrium evolution of hydrogen and helium ionic species. The gas is allowed to interact with the CMB through Compton cooling, with the XRB through Compton heating, and with the time-dependent QSO ionizing background as computed by HM96.

It is widely believed that the diffuse XRB is due to the summed emission from AGNs (see Fabian \& Barcons 1992 for a review). There is insufficient information, however, to uniquely infer from the data the evolution with cosmic time of the hard $\mathrm{X}$-ray volume emissivity. According to the findings of Boyle et al. (1994) from ROSAT data (see also Page et al. 1996), the model that best fits the cosmological evolution of soft X-ray selected AGNs in an Einstein-deSitter universe is one in which the comoving volume emissivity increases as $(1+z)^{3.15}$ for $z<1.6$ (in qualitative agreement with the evolution of the QSO optical luminosity function), and remains roughly constant at higher redshifts, up to $z \sim 4$ (Miyaji, Hasinger, \& Schmidt 1998). Assuming that these simple prescriptions also describe the evolution of hard X-ray sources, the energy density of the XRB then increases approximately as $U_{X} \propto(1+z)^{4}$ at low redshifts, and as $(1+z)^{2.5}$ at $z>1.6$. To account for the existence of a high redshift cut-off, we will parameterize the evolution of the $\mathrm{X}$-ray energy density as

$$
U_{X}(z)=U_{X}(0)(1+z)^{4} \exp \left(-z^{2} / z_{c}^{2}\right) .
$$

Figure 1 shows the ensuing Compton heating rate per baryon as a function of redshift for $z_{c}=5$, compared with the photoionization heating rate from optically-selected quasars. Figure 2 shows the thermal history of an IGM with $\omega_{b}=0.019$ (Burles and Tytler 1998) for two cosmological models: an Einstein-deSitter universe and a spatially flat universe dominated by a cosmological constant with parameters close to those inferred from Type Ia supernovae and cosmic microwave background anisotropies (see Efstathiou et al. 1999, and references therein). In both cases, we assume a photoionizing background as computed by HM96 and a Hubble constant of $h=0.65$ (see 
Freedman et al. 1998). The dotted lines show the temperature evolution if the X-ray background is ignored, as has been assumed in all previous work. The solid lines include Compton heating by the XRB according to the model of equation (11). Including the X-ray background with a redshift cut-off parameter of $z_{c}=5$ causes the temperature of the IGM to rise to $1.4 \times 10^{4} \mathrm{~K}$ at $z=4$ and $1.1 \times 10^{4} \mathrm{~K}$ at $z=3$. The IGM temperatures are even higher in the $\Lambda$-dominated model. These results are extremely insensitive to the amplitude of the photoionizing background and relatively weakly dependent on $\omega_{b}$ and $h$. They are, however, sensitive to the assumed redshift evolution of the $\mathrm{X}$-ray background and to the hard $\mathrm{X}$-ray spectrum at photon energies above $50 \mathrm{keV}$, which is poorly known but dominate the heating rate.

The results of Figure 2 and the numerical simulations of Theuns et al. (1999) suggest that heating of the IGM by the hard XRB may well be able to account for the $b$-parameter distribution of the Ly $\alpha$ forest. Furthermore, our results suggest that the observed $b$-parameter distribution may provide a useful probe of the evolution of $\mathrm{X}$-ray diffuse radiation at high redshift. Compton heating will also tend to flatten the equation of state of the intergalactic gas, since the Compton heating per unit mass is independent of density. However, for our model with $z_{c}=5$, this effect is small, even for low density regions.

\section{Discussion}

We have shown that Compton heating of electrons by hard X-ray background photons provides an important heating source of the IGM. For plausible parameters, heating by the XRB will be comparable to photoelectric heating at redshifts $\gtrsim 2$. This effect has been ignored in most numerical simulations of the Ly $\alpha$ forest, yet it can have a significant impact on the temperature of the IGM, raising it to temperatures as high as $\sim 1.5 \times 10^{4} \mathrm{~K}$ at redshifts $z \gtrsim 3$.

The increase in IGM temperature caused by this effect may explain why the Ly $\alpha b$-parameter distributions derived from numerical simulations (Theuns et al. 1998b; Bryan et al. 1999) are narrower than those observed. Theuns et al. (1999) have previously suggested that the temperature of the IGM can be raised by invoking a high baryon density in a universe with low total matter density. However, the density required, $\omega_{b} \gtrsim 0.025$, is well above the value implied by primordial nucleosynthesis and the measurements of the deuterium abundances from quasar spectra $\left(\omega_{b}=\right.$ $0.0193 \pm 0.0014$, Burles and Tytler 1998). Compton heating by hard X-ray photons may help to resolve this discrepancy. The origin and main production epoch of the XRB are still largely unsolved problems. The $b$-parameter distribution of the Ly $\alpha$ lines could provide important information on the evolution of $\mathrm{X}$-ray background radiation at high redshifts.

Finally, it is interesting to note that, as another consequence of Compton heating of the IGM, there may exist an X-ray proximity effect, in analogy with the usual UV proximity effect (e.g. Bechtold 1994 and references therein). This could result in a hotter IGM, and consequently

broader Ly $\alpha$ lines, in the immediate vicinity of a long-lived X-ray emitting quasar (lifetime $\gtrsim$ the 
Compton heating timescale).

We have benefited from useful discussions with G. Bryan, A. Meiksin, J. Miralda-Escudé, M. Rees, and T. Theuns. We are especially grateful to Jordi Miralda-Escudé for pointing out the importance of relativistic corrections at high redshift. Support for this work was provided

by NASA through ATP grant NAG5-4236, and by NSF through grant PHY94-07194(PM). GPE thanks PPARC for the award of a Senior Fellowship.

\section{REFERENCES}

Abel, T., \& Haehnelt, M. G. 1999, submitted to ApJ (astro-ph/9903102)

Bechtold, J. 1994, ApJS, 91, 1

Blumenthal, G. R. 1974, ApJ, 188, 121

Boldt, E. 1987, Phys. Rep., 146, 215

Boyle, B. J., Shanks, T., Georgantopoulos, I., Stewart, G. C., \& Griffiths, R. E. 1994, MNRAS, 271,639

Bryan, G. L., Machacek, M., Anninos, P., \& Norman, M. L. 1999, ApJ, in press (astro-ph/9805340)

Burles, S., \& Tytler, D. 1998, ApJ, 499, 699

Cen, R., Miralda-Escudé, J., Ostriker, J. P., \& Rauch, M. 1994, ApJ, 437, L83

Davé, R., Hernquist, L., Katz, N., \& Weinberg, D. 1999, ApJ, 511, 521

Efstathiou, G., Bridle, S. L., Lasenby, A. N., Hobson M. P., \& Ellis, R. S. 1999, MNRAS, in press (astro-ph/9812226)

Fabian, A. C., \& Barcons, X. 1992, ARA\&A, 30, 429

Freedman, J. B., Mould, J. R., Kennicutt, R. C., \& Madore, B. F. 1998, preprint (astro-ph/9801080)

Haardt, F., \& Madau, P. 1996, ApJ, 461, 20 (HM96)

Haehnelt, M., \& Steinmetz, M. 1998, MNRAS, 298, L21

Hernquist, L., Katz, N., Weinberg, D., \& Miralda-Escudé, J. 1996, ApJ, 457, L51

Hui, L., \& Gnedin, N. 1997, MNRAS, 292, 27

Kappadath, S. C., et al. 1996, A\&AS, 120, 619

Kinzer, R. L., Jung, G. V., Gruber, D. E., Matteson, J. L., \& Peterson, L. E. 1997, ApJ, 475, 361

Kribs, G. D., \& Rothstein, I. Z. 1997, Phys. Rev. D., 55, 4435

Madau, P. 1992, ApJ, 389, L1

Miralda-Escudé, J., Cen, R., Ostriker, J. P., \& Rauch, M. 1996, ApJ, 471, 582 
Miralda-Escudé, J., \& Rees, M. J. 1994, MNRAS, 266, 343

Miyaji, T., Hasinger, G., \& Schmidt, M. 1998, preprint (astro-ph/9809398)

Nath, B. B., Sethi, S. K., \& Shchekinov, Y. 1999, MNRAS, 303, 1

Page, M. J., et al. 1996, MNRAS, 281, 579

Shull, J. M., \& Van Steenberg, M.E. 1985, ApJ, 298, 268

Theuns, T., Leonard, A., \& Efstathiou, G. 1998a, MNRAS, 297, L49

Theuns, T., Leonard, A., Efstathiou, G., Pearce, F. R., \& Thomas, P. A. 1998b, MNRAS, 301, 478

Theuns, T., Leonard, A., Schaye J., \& Efstathiou, G. 1999, MNRAS, in press (astro-ph/9812141)

Zhang, Y., Anninos, P., \& Norman, M. L. 1995, ApJ, 453, L57

Zhang, Y., Meiksin, A., Anninos, P., \& Norman, M. L. 1998, ApJ, 495, 63 


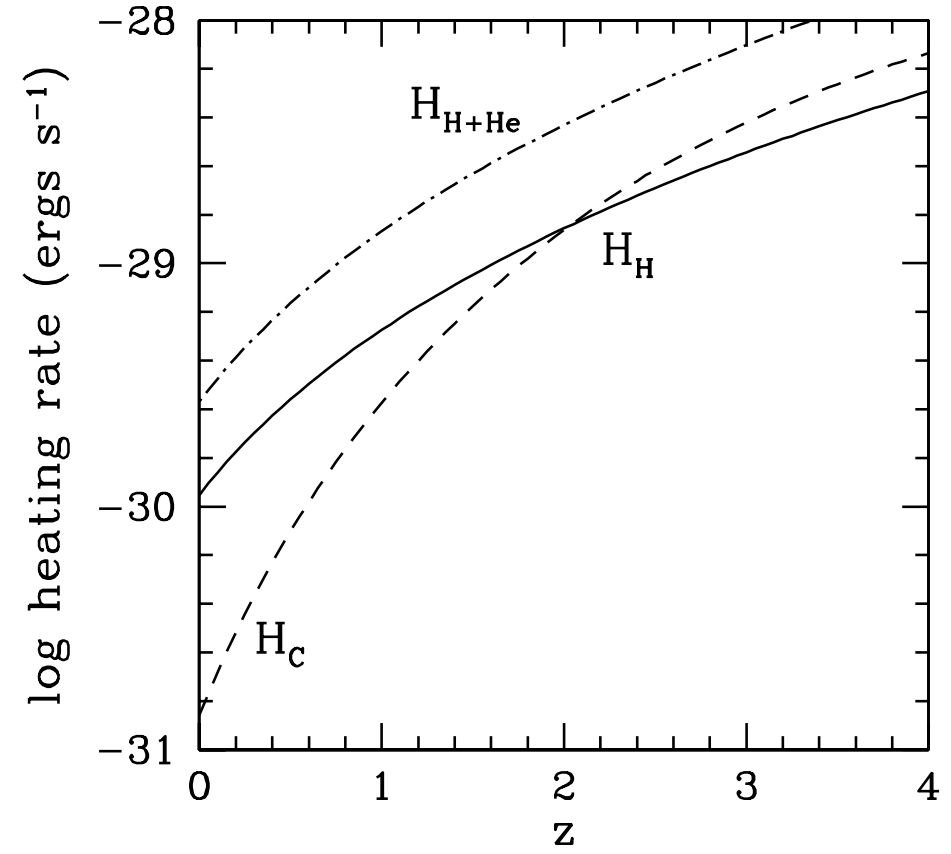

Fig. 1.- Compton (dashed line) and photoionization (solid line: $\mathrm{H}$ only; dash-dotted line: $\mathrm{H}+\mathrm{He}$ ) heating rates per baryon as a function of redshift. The rates have been derived assuming ionization equilibrium in an IGM with $\omega_{b}=0.019$, the HM96 UV radiation field, the XRB evolution described in the text (with $z_{c}=5$ ), and the corresponding temperature evolution shown in Figure $2 b$. 


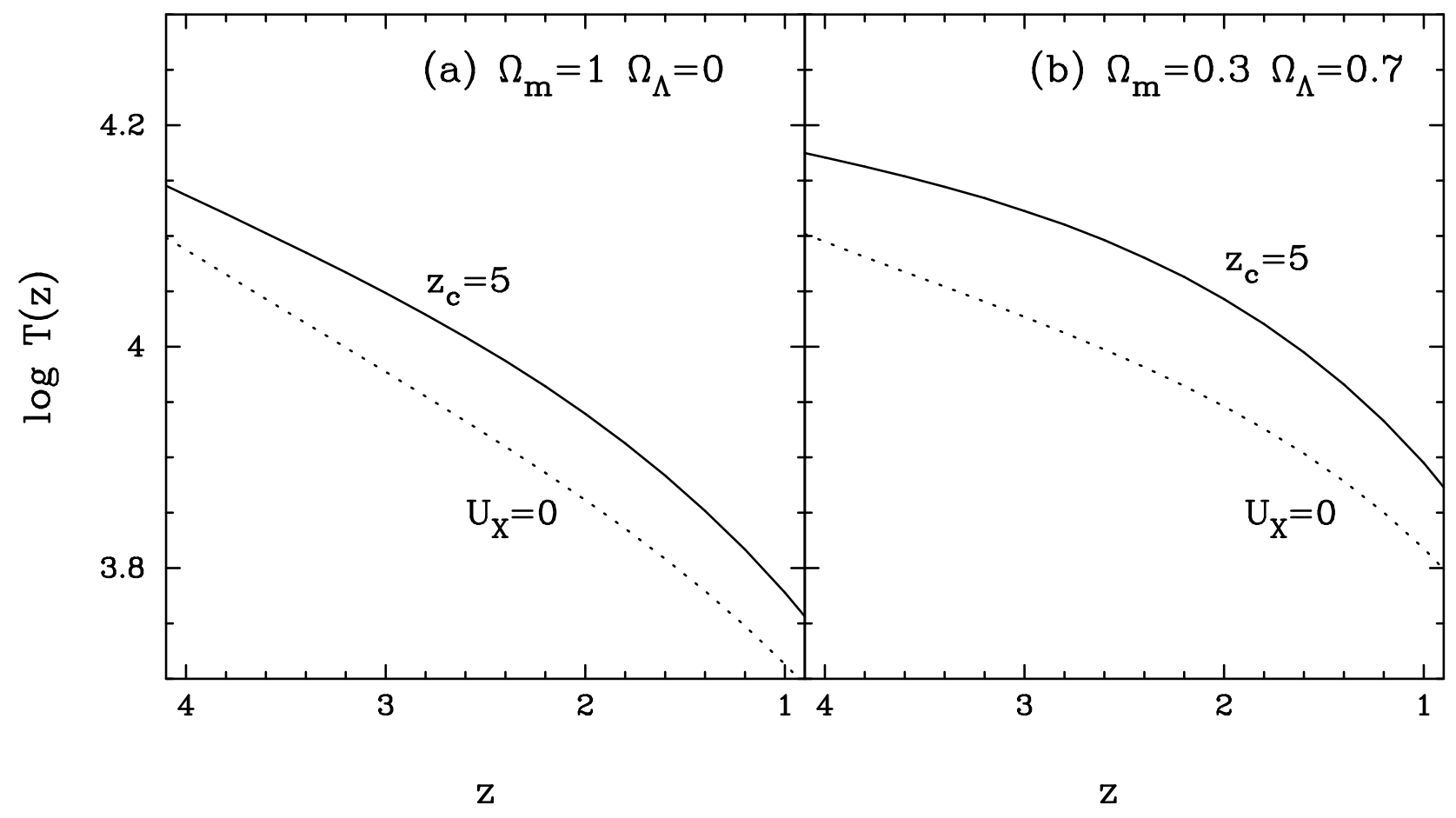

Fig. 2. - Thermal history of a uniform intergalactic medium for two spatially flat cosmologies. The dotted lines show the temperature evolution when the $\mathrm{X}$-ray background is ignored and the only heating source is the quasar UV background as computed by HM96. The solid lines show the temperature evolution when the $\mathrm{XRB}$ is included with redshift evolution as given by equation (11) for $z_{c}=5$. We have assumed $\omega_{b}=0.019$ and $h=0.65$. 